\title{
A New Conceptual Modelling Method for Vehicle Subframe to Evaluate Dynamic Performance of Structures at Conceptual Design Stage
}

\author{
S. Azaripour and A. Masoumi* \\ School of Mechanical Engineering, College of Engineering \\ University of Tehran, Tehran, Iran \\ *Email: amasomi@ut.ac.ir
}

\begin{abstract}
In recent years, the reduction of noise and vibrations caused by the road or internal components of vehicles have been a significant factor in the satisfaction and comfort of the occupants of cars. Therefore, to minimize these vibrations, the dynamic behaviour of the components of the vehicle should be considered in detail to reduce their level by setting useful parameters on structures. Nowadays, the conceptual model method can be regarded as a suitable alternative to the fundamental sophisticated computational techniques in measuring the vibration of vehicle components. Accordingly, the advanced finite element (FE) model examined for the subframe structure under the vehicle engine, and its conceptual model is developed by the one-dimensional beam elements to justify the dynamic behaviour of the subframe. The results of the experiments in the laboratory as well as the advanced subframe model are adapted, and the integrity of the natural frequencies and the mode shapes at low frequencies represented comprehensively. The result of the subframe concept model compared to the experimental model and computer-aided-engineering (CAE) model showed that the modal assurance criterion (MAC) is above 0.75 for the first four mode shapes of subframe structure and is above 0.9 for first and fourth mode shapes, and also the error percentage of natural frequency is lower than $8 \%$. Therefore, for the analysis of the subframe performance in noise, vibration, and harshness $(\mathrm{NVH})$ domain, the presented model could be considered in the conceptual phase design to reduce the solution time significantly.
\end{abstract}

Keywords: Conceptual model; vehicle structure; advanced CAE model; subframe

\section{INTRODUCTION}

The conceptual modelling is the initial step of design in which models are used to evaluate the behaviour of the structure and body of the vehicle when the details of the vehicle body are not added, yet. These models can reduce the required time for the dynamic analysis of the vehicle structure considerably. In the event of the evolution of existing structures, the engineers attempt to update the conceptual models of each composition according to the resulting changes. Therefore, the error rates are minimised, and the desired result maintained.

Due to problems for simulations, general and straightforward models with low computational volume considered for the first time in the industry. Gradually with the advancement of the computer simulation equipment, these models developed and covered all sections in detail. Because of the predicting power of simple models, they have regained their importance in the conceptual design phase and emerged again in the industry $[1,2]$. In general, conceptual modelling is divided into two categories. In the first category of conceptual modelling which is called optimisation of design topology; the whole vehicle 
body is considered as a design parameter and is modelled with three-dimensional elements. In the next step, the presence or absence of components in a different situation is discussed with the help of the results of the optimisation and creating a specific objective function. Usually in the NVH domain of the static hardness of the vehicle body[3]. For example, Wang et al. [4] considered enhancing the hardness of the vehicle body using optimisation techniques in the finite element.

In this study, the torsional or flexural principal mode shape frequency represents the hardness of the vehicle body. Gur et al. [5] analysed damping properties of the carbon fibres composite parts in B-pillar to evaluate dynamic performance in vehicle design and show the result of mode shapes and modal damping. Raasch [6] considered a rectangular section for all beam-shaped parts modelled with one-dimensional elements along with it concerning the optimisation results of the surfaces. Also, the joints shaped as T-shaped and multi-channel sections, and panels were constructed with significant two-dimensional elements and connected to the beams with rigid non-dimensional elements at specific locations. Zou et al. [7] simplified the passenger car seat to a cantilever beam physical model and analysed seat vibration with an effective seat modal test method to enhance the vehicle dynamic comfort. You et al. [8] developed a model based on the same principle. In their model, in addition to optimising of the beam sections and the size of the body components with the vibration analysis criterion, it is possible to change the position of different structural elements to maximize the body structure performance. The process of optimising the design of the car structure and the design of the components of the body structure with a lower weight model has been studied by Lee et al. [9]. In this research, the specification of the vehicle has been optimised for the maximum stiffness and minimum body weight.

In the second category of conceptual modelling which is carried out based on the previous model, the information in the detail-based model of the preceding vehicle is very useful in conceptual modelling. Fard [10] provided conceptual modelling only with the help of one-dimensional elements. In this model, only the main components of the vehicle body structures that constitute the principal framework are in the conceptual model. Sung et al. [11] examined conceptual modelling with the use of 1-D elements. In their model, the components of the overall structure of the body and auxiliary parts were considered to evaluate the effects of other surfaces. Long [12] conducted research that linked the application goals of the vehicle with the connections, and in fact, the geometry of the model was determined according to the bonds' arrangement. Lee et al. [13] studied the impact of joint stiffness. They initially calculated the equivalent one-dimensional elements for the main components of the vehicle using advanced model information and built their conceptual model using only the bar elements by ignoring the deformation of the primary connections. Kiani et al. [14] introduced a new method for the evaluation of the structural performance of the vehicle through the stiffness of the joints. They proposed the sensitivities of the vibration responses to the joint stiffness. Moroncini et al. [15] considered the conceptual model derived from the shell and beam elements. They optimised the thickness of the sheet to control the vibration of the vehicle under study. Also, they were able to reduce the bodyweight of BMW. Mundo et al. $[16,17]$ proposed replacement methodology based on the reduced beam and joint modelling approach which involves a geometric analysis of beam-member cross-sections and a static analysis of joints. They identified the beam centre nodes and computed the equivalent beam properties. They also analysed a simplified model of a joint that connects three or more beam-members through a static reduction of the detailed joint FE model. Tebby et al. $[18,19]$ used the beam elements to create a structural model of the vehicle. Their approach used a numerical FE method and can determine unknown deflections and reaction forces as well as the internal loading on each member. 
Marburg et al. [20] considered design optimisation of the geometry of a vehicle hat-shelf. They decreased the vehicle interior noise due to three different excitations for two cases of fluid damping. Zhu et al. [21] proposed a study on the lightweight design of the automotive front-body structure based on robust optimisation, considering the variation in design variables including sheet gauge and yield limit of materials. Gundogdu [22] presented an optimisation of a four-degrees-of-freedom quarter car seat and suspension system using genetic algorithms to determine a set of parameters to achieve the best performance of the driver. Zargar et al. $[23,24]$ proposed a conceptual modelling method in which beams and panels of the structure modeled as simplified beam elements. They considered an experimental test and advanced $\mathrm{CAE}$ analysis to measure the dynamic characteristics of the wheelhouse.

In the present research, a new improvement on the conceptual model has been developed based on modifications recommended by Zargar et. al.[23] and Mundo et. al.[17]. This method is implemented in the subframe structure of a sedan. Then, the results of the simulation of the structure and the experimental modal analysis are compared with the results of the proposed model to assess the exactness and execution of this strategy. This demonstration can be utilised for distinctive parts of the body in vehicles owing to the capacity to broaden of components utilised to simplify structures and assigning a different type of beams to produce a new conceptual model in every variegated single part. Using this new conceptual model essentially decreases vibration examination time. Also, this approach can provide the natural frequencies and mode-shapes to the designer with much less computation and complexity than commonly used methods and it allows the design flaws to resolve in the initial phases of design.

\section{EXPERIMENTAL METHOD}

The modal analysis experiment has been used to obtain modal parameters of a subframe. The primary purpose of collecting these modal parameters is to create, compare, and modify an FE model of the subframe. This structure is one of the leading sources of generating and transmitting vibration to the vehicle. Therefore, the prediction of its behaviour is a fundamental issue. When the subframe is excited at its resonance frequencies, it vibrates in particular forms called mode shape. These experiments include excitation by a shaker with an electrodynamic head and measuring frequency response functions at some specific points on the subframe.

A modal analysis test is conducted to determine the original mode shapes and their corresponding frequency for the subframe structure of a sedan car. The tested set, as shown in Figure 1, includes subframe structures, holders and excitation mechanisms with the required hardware and software. The subframe structure is suspended using several ropes to obtain its dynamic properties in free conditions. This type of suspension allows the structure to move freely in six directions. Although different methods are used to create free boundary conditions in the modal analysis, according to the available facilities the elastic ropes were preferred to other techniques. Elastic ropes can maintain the rigid position of the frame vertically and tolerate its weight perfectly. 


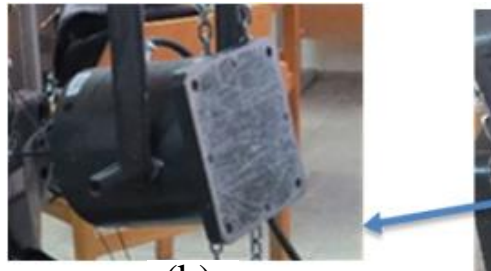

(b)

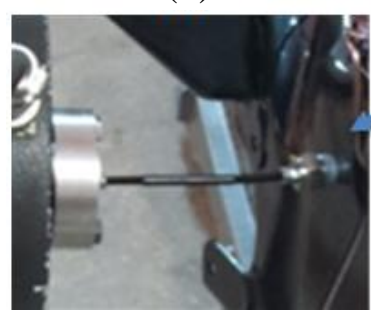

(c)

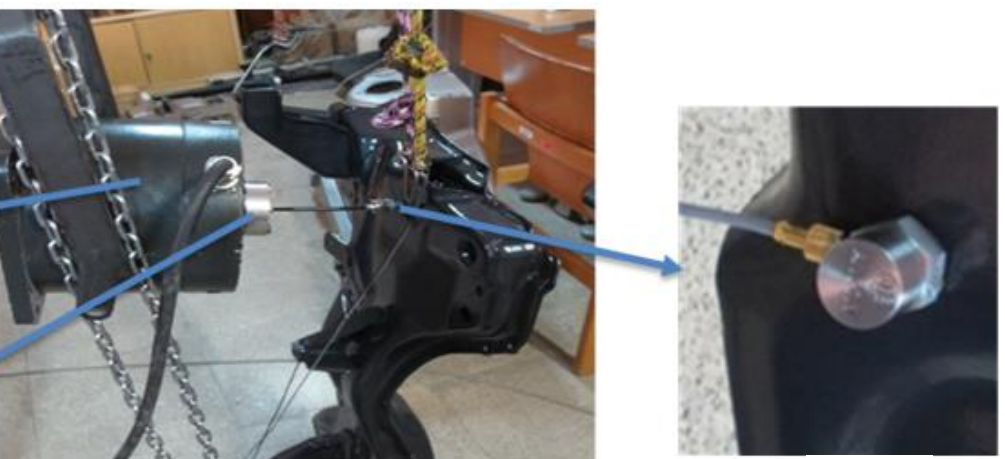

(d)

Figure 1. General view of the experimental setup: (a) subframe structure assembly (b) shaker (c) stringer with a force transducer (d) accelerometer sensor.

Pulse software, one-axial accelerometer, plastic stringer, loading transducer (8200+ 2647), a data logger (B \& K 356), amplifier (B \& K 2786), and electromagnetic shaker (B \& $\mathrm{K} 4808$ ) were the equipment needed to carry out tests on the suspended structure. Power transducers were other types of sensors used in vibration analysis. Similar to the accelerometer, a piezoelectric force transducer produces an output signal in the form of a current or voltage proportional to the force applied to the transducer and this signal can be affected by alternations in mechanical forcing, thermal gradient, the shape of plates used in the piezoelectric structure [25-28]. Unlike the accelerometer, the transducer does not have masses connected to the sensor element. The power transducer will produce output if the sensor sector is compressed or pulled. In the vibrator test, the power transducer should be placed between the vibrator and the surface of the structure. Therefore, they were fixed in the upper part of the subframe structure by an adhesive.

In the second stage, the laboratory equipment was prepared to perform the modal analysis. In the subframe modal test, a fixed shaker test for extraction mode shapes and structural responses of the subframe was used. Finally, the received signals from the accelerometer and the shaker connected to the inputs of the data-processing machine [29]. Pulse software used for data processing and the necessary settings were made to improve the data quality. This method is beneficial for obtaining frequency response function (FRF) charts, which represent the system behaviour, and can be used to extract behaviour the system and more importantly the natural frequencies[30-32].

By performing the experimental modal tests of the subframe, the FRF charts were obtained by the electromagnetic shaker input at different points and the accelerometer output at a fixed position which display a row of the frequency response examples of which are represented in Figure 2. For instance, the first mode shape has occurred near a frequency of $100 \mathrm{~Hz}$. The peak frequencies in the FRF graphs represent the typical frequencies of the subframe structure, and the mode shapes of each structure can be easily extracted from the resonance slopes of the FRF graphs. In fact, by choosing one of the natural frequencies, the acceleration amplitude of all the FRF graphs at that frequency with the correct phase is defined as the specific vector of the degree of freedom and its mode shape is presented by the eigenvector of a natural frequency. 


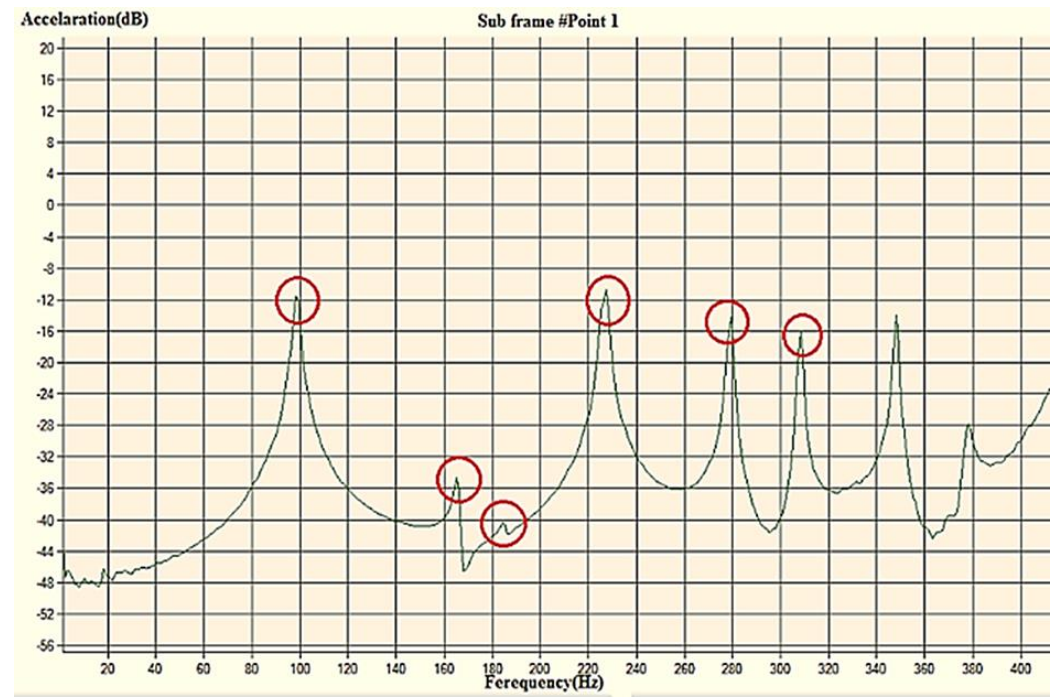

(a)

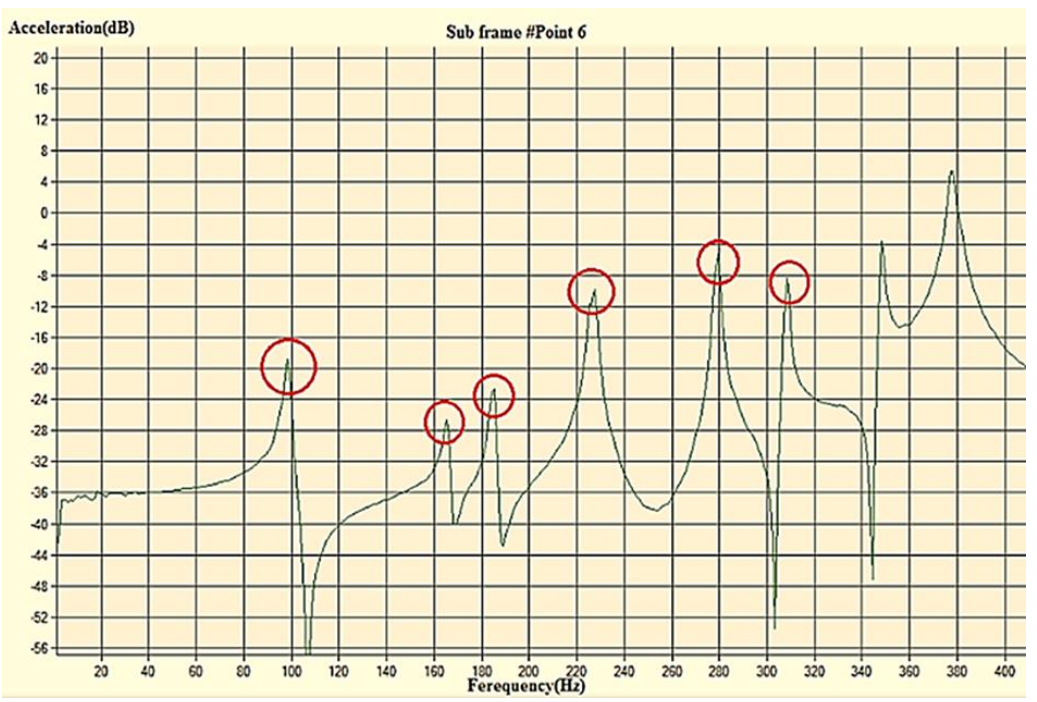

(b)

Figure 2. Frequency response function diagram at (a) point 1 and (b) point 6.

\section{Conceptual Modelling}

In the conceptual modelling, the advanced model of subframe structural engineering calculations consisting of a mass of one dimensional, dual force and beam, two dimensional triangular and quadrilateral shells and lines were modelled by CBAR beam shape elements and multiple weld points were developed by CWELD elements. In the elements' layout, it was attempted to match with the structure by the central, fundamental points (such as the connecting points of the handles, the frame of the subframe, the corners of the panels connecting to each other), or the ends representing the main subframe structure.

In this process, the upper and lower sheet panels were modelled in a conceptual model using one-dimensional beam elements, and then they were linked to other structural components, and the final model was constructed. It was attempted to use 1-D elements for beam and available panels in the upper and lower panels of the subframe for more simplification and generalizability in the mode shapes. Beam element (e.g., PBEAM in 
Nastran software [33]) and rigid elements (e.g., RBE2 in Nastran software [33]) were used to model the parts of the subframe structure.

The final model was developed using 128 CBAR elements. During these steps, it was attempted to replace the sections with the most similarities to the main structure properties by the trial and error to reduce the chance of failure. Due to the cross-sectional deformation in different parts, a separate cross-section should be defined for each one-dimensional beam to provide features similar to the modelled section. The use of beam components with small sections in this modelling has led to consider the effects of heterogeneity due to joints, curvatures of the primary model in different parts. The advantage of CBAR elements which were used in this modelling was that they can be defined with the help of their cross-sectional features. This makes these elements to tolerate bending and torsional forces along with the tensile and stress tolerance.

\section{Numerical Model}

For subframe modelling, the material properties were entered in the software as given in Table 1. It should be noted that the CATIA software was used to design the surface of the structure precisely and then the HyperMesh software applied for meshing. The final analysis was done with the help of NASTRAN software.

The subframe was geometrically modelled in exact details because the subframe's dynamic behaviour highly depends on its form and in case of failure to observe small circumstances, otherwise, it will not be possible to get the right model. The geometry and shape of the plates have a more significant impact on the model's hardness, and therefore on the resonance frequency of the model. Another critical factor that affects the hardness of the subframe is the precise modelling of joining the pieces of arms and plates to each other. The upper and lower plates connected by the weld point that the RBE3 element should be defined by the NASTRAN software for their modelling.

Table 1. Material properties of subframe structure.

\begin{tabular}{lcc}
\hline Material specifications & Matric system & Hyper Mesh system \\
\hline Young's modulus, E & $210 \mathrm{GPa}\left(\mathrm{N} / \mathrm{m}^{2}\right)$ & $2.1 \mathrm{e} 8 \mathrm{mN} / \mathrm{mm}^{2}$ \\
Poisson coefficient, Nu & 0.3 & 0.3 \\
\hline Density, $\rho$ & $78000 \mathrm{~kg} / \mathrm{M}^{3}$ & $7.8 \mathrm{e}-6 \mathrm{~kg} / \mathrm{mm}^{3}$ \\
\hline Structural damping, GE & 0.05 & 0.05 \\
\hline
\end{tabular}

Also, as the $\mathrm{CO}_{2}$ weld line is used to connect side accessories to two central plates, the RBE2 element in NASTRAN software was used to model it. For full meshing of the structure, elements with a $9 \mathrm{~mm}$ dimension were used in the form of a quadrangle and a triangle that is introduced as PSHELL in the NASTRAN software. This kind of meshing covers all surfaces with much complexity. The meshed subframe is illustrated in Figure 3.

After validation of the FE model, the convergence test of meshing must be satisfied. In this research, a grid-independent technique is used for determination of the grid convergence. Six different elements were applied to the structure and the natural frequencies between the same modes of these four sets will determine the convergence. Figure 4 shows the natural frequency between the same modes when six different elements were applied in the FE model of the subframe. It can be observed that for these four sets the difference is less than $1.7 \%$ which is an acceptable threshold. 


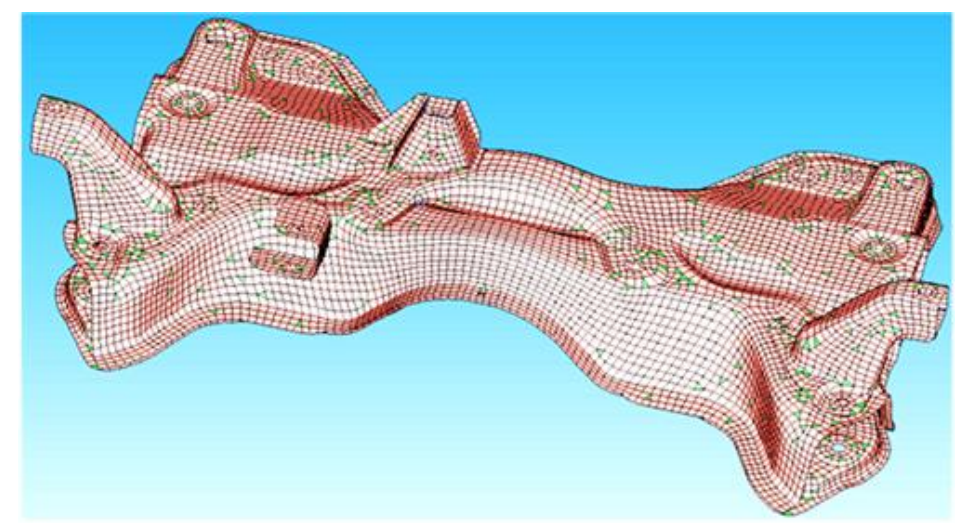

Figure 3. General view of grid mesh.

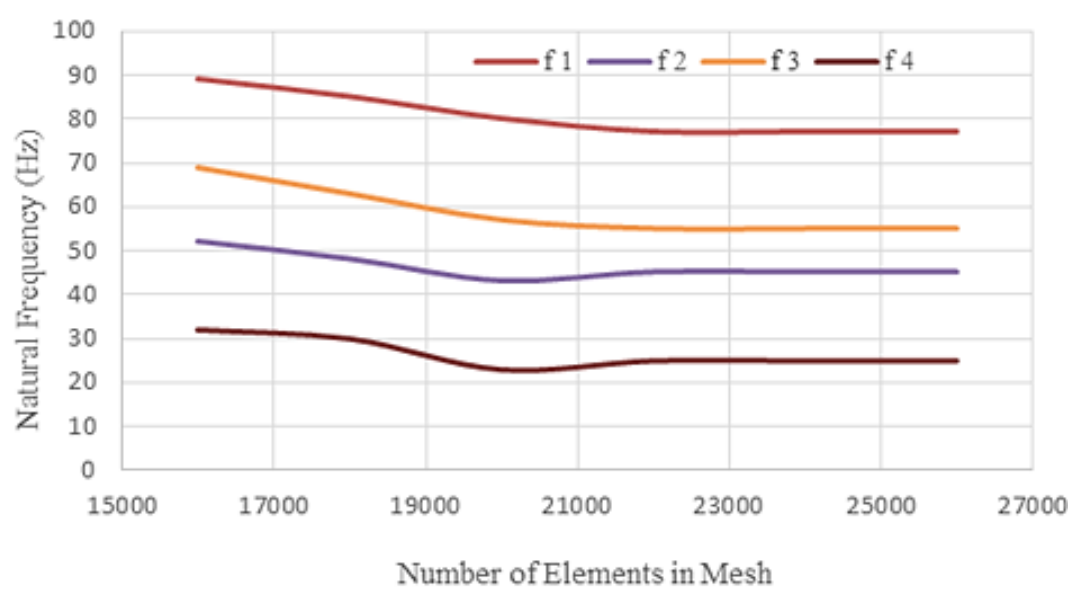

Figure 4. The convergence diagram.

In this type of modelling, the plate between the two layers of the sheets in both upper and lower parts is defined as the middle level. The welded points determined by specific coordinates. In these points, the solid object is described as a square or cylindrical shape, the diameter of which is considered between the elements of meshing. This rigid component is located and limited between the two middle plates forming the way. A total of 44 weld points and 18 weld lines have been used in the above modelling, and the weld joints are shown in Figure 5.

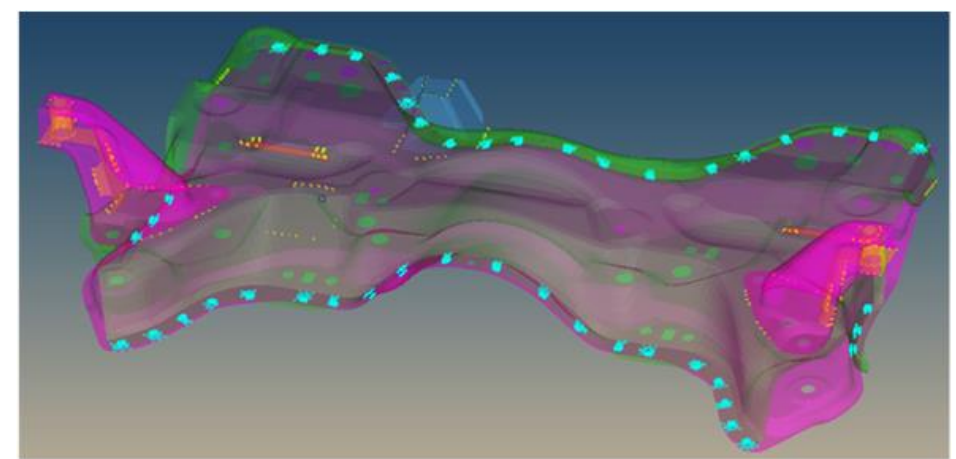

Figure 5. General view of weld spots on the subframe. 
It should be noted that there are usually several thousand degrees of freedom in the frequency analysis of a structure. Only in such cases, some initial natural frequencies and the corresponding modes are applied and studied. The NASTRAN solution method for eigenvalue problems is the Block Lanczos method with the repeated invertible matrix method and the introduction of the shift parameter, which is a conventional method for dynamic analysis of structures.

\section{RESULTS AND DISCUSSION}

The information on the conceptual model and the CAE model of the subframe is shown in Table 2. Also, the developed conceptual model is presented in Figure 6. In order to compare and validate the model, first the fundamental and underlying features in the dynamic domain are compared, and if the result is appropriate, the comparison criterion will go one step forward, and relatively more complex features will be used for a more accurate comparison. The natural frequencies of the structure are the main features in the dynamics domain. Therefore, the first and most straightforward comparison criterion is the natural frequencies of the two models.

Table 2. Comparison of detailed information for CAE and conceptual model.

\begin{tabular}{lclc}
\hline CAE model & numbers & Conceptual model & numbers \\
\hline Spot Welds & 44 & Beam elements & 128 \\
Bead Welds & 18 & Solid elements & 32 \\
\hline Shell elements & 13288 & Concentrated mass & 21 \\
\hline Mass & 12.585 & Mass & 12.50 \\
\hline Time of solution & $5.50 \mathrm{~min}$ & Time of solution & $11.03 \mathrm{sec}$ \\
\hline
\end{tabular}

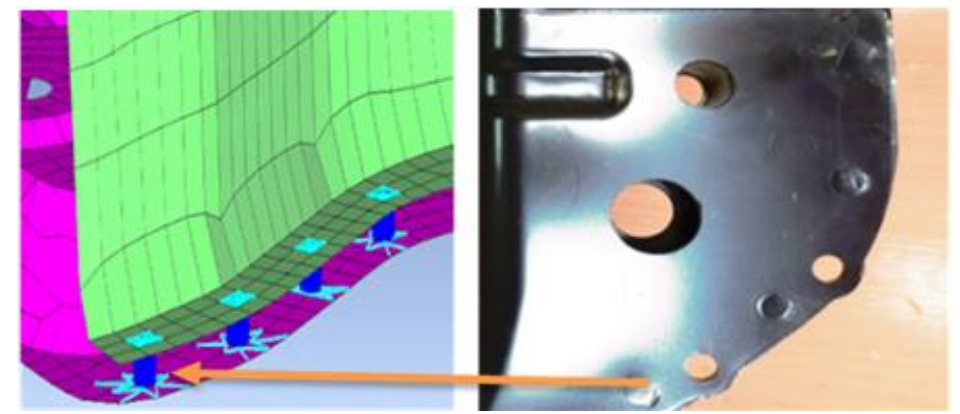

(a)

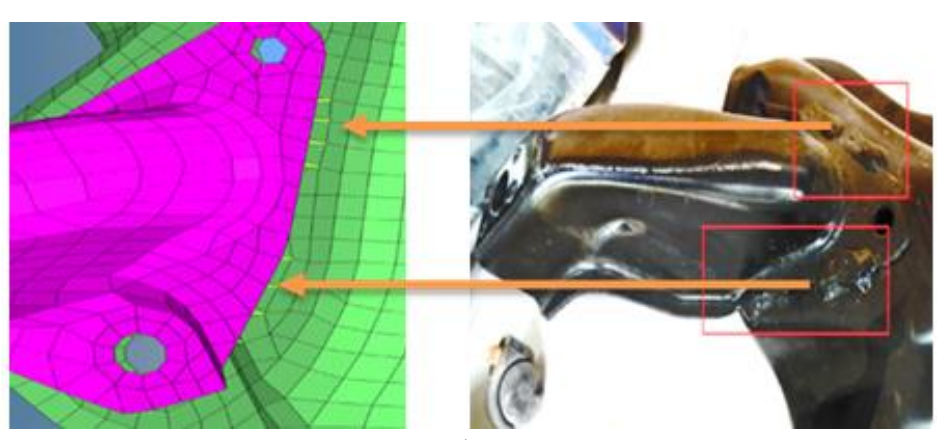

(b)

Figure 6. Representation of welds on subframe: (a) spot welds and (b) bead welds. 


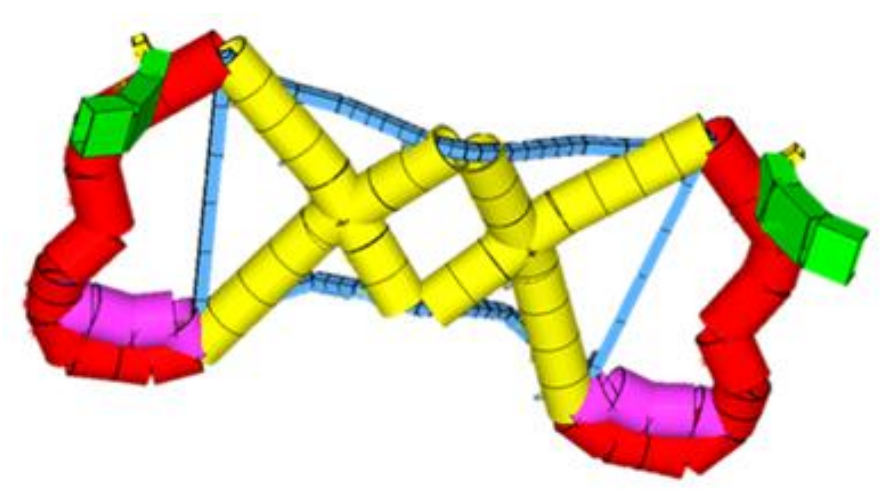

Figure 7. Final conceptual modelling of subframe structure.

The expression of the natural frequency numbers obtained from conceptual and the advanced numerical models paves the way for the initial validation of the results, but since the comparison should be conducted, it is better to deal with this issue by the error rate method. The error rate of the frequencies is calculated according to Eq. (1). The results for the first four frequencies of the subframe are listed in Tables 3.

$$
\Delta \%=\left|\frac{\left(\omega_{c}-\omega_{a}\right)}{\omega_{a}}\right| \times 100
$$

Table 3. Comparison of subframe resonant frequencies between the conceptual model and advanced model in different mode numbers.

\begin{tabular}{lccc}
\hline Mode number & Advanced model $(\mathrm{Hz})$ & Conceptual model $(\mathrm{Hz})$ & Error rate, $\Delta \%$ \\
\hline 1 & 99.1 & 100.6 & 1.41 \\
2 & 196.5 & 203.1 & 3.36 \\
\hline 3 & 203.7 & 211.7 & 3.93 \\
\hline 4 & 235 & 233.4 & 0.68 \\
\hline
\end{tabular}

After comparing the natural frequencies, it is time to examine the mode shapes derived from these frequencies. There are various methods for analysing the similarity of the mode shapes graphically and mathematically. Graphical means of comparing two mode shapes are swift and straightforward. A simple way is to put the graphical representation of two modes besides each other and examine the difference between them visually. Although this technique is very convenient and it seems very capable for simple models with only a few degrees of freedom, but it cannot be suitable for intricate models, and its main drawback is the difficulty in interpreting the difference in the two models. Therefore, using a reasonable mathematical criterion is very helpful $[34,35]$.

Contrary to the graphical methods to compare the eigenvectors, mathematical methods lead to significant statistical and qualitative information for the difference between the two models. In this research, MAC was selected as a mathematical method for comparing the vectors. The comparison of the mode shapes with MAC is obtained by computing the relationship, which represents a number between zero and one after calculation. In the case of two identical mode shapes, the resulting number is one, and this result indicates that the two eigenvectors are dependent and linear. On the other hand, if the resulting number will be zero, the finding suggests that the eigenvectors are independent and linear or the natural 
modes are orthogonal. In Eq. (2), $\phi_{i}$ is the mode shape eigenvectors to be compared with $\phi_{j}$ $[10,36]$. By examining and comparing the results presented in Table 4 , and given the simplifications in case of slight difference in the coordinates of the nodes used in the original and conceptual models in the one-dimensional equivalent elements instead of twodimensional, the MAC also yields acceptable results.

$$
\operatorname{MAC}_{i j}=\frac{\left(\left\{\phi_{i}\right\}^{T}\left\{\phi_{j}\right\}\right)^{2}}{\left(\left\{\phi_{i}\right\}^{T}\left\{\phi_{i}\right\}\left\{\phi_{j}\right\}^{T}\left\{\phi_{j}\right\}\right)}
$$

Table 4. Comparison of subframe resonant frequencies and MAC.

\begin{tabular}{lcccc}
\hline Mode number & Experimental model $(\mathrm{Hz})$ & Simulation $(\mathrm{Hz})$ & Error, $\Delta \%$ & MAC \\
\hline 1 & 97 & 99.1 & 2.16 & 0.91 \\
\hline 2 & 182 & 196.5 & 7.96 & 0.81 \\
\hline 3 & 188 & 203.7 & 8.35 & 0.75 \\
\hline 4 & 229 & 235 & 2.62 & 0.90 \\
\hline
\end{tabular}

Accordingly, the effectiveness of the proposed method for the conceptual design of the vehicle is confirmed. The results of the analysis show the interpretive match of the mode shapes of the structure. The MAC numbers greater than 0.9 is a perfect match, numbers between 0.8 and 0.9 are appropriate to match, numbers between 0.7 to 0.8 is an acceptable event, and the numbers below these intervals are considered unacceptable by the analysts. These modes have occurred after six rigid modes of subframe structures and are among the essential vibrational modes for dynamic analysis. In the first torsion mode of the structure entirely matches for the conceptual model in this criterion with the values of 0.91 and 0.9 in the fourth mode. There is a smaller MAC number in the second and third modes the reason for which is in the occurrence of the hybrid and local modes of the structure. In other words, the appearance of different mode shapes in a frequency reduced the matching to the first and fourth modes and reduced the MAC number slightly.

Local modes usually are created in any structure, and they occur in different frequency ranges depending on its physical and structural properties. Of course, it is noteworthy to note that the first and fourth modes are significant for modal analysis because they occur individually rather than locally and influence the structural behaviour by their effects on the initial frequencies. Therefore, the validity of the model is accepted concerning the above numbers. The mode shapes of these models were shown in Figure 8.

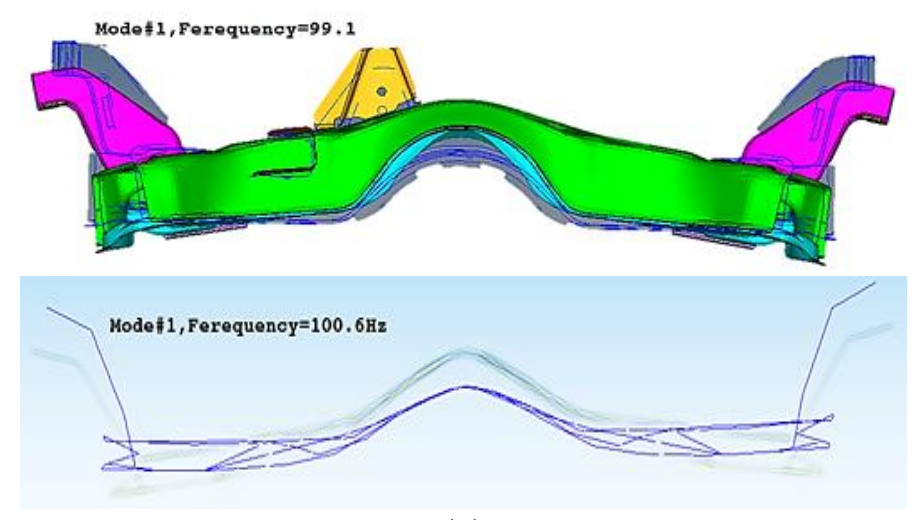

(a) 


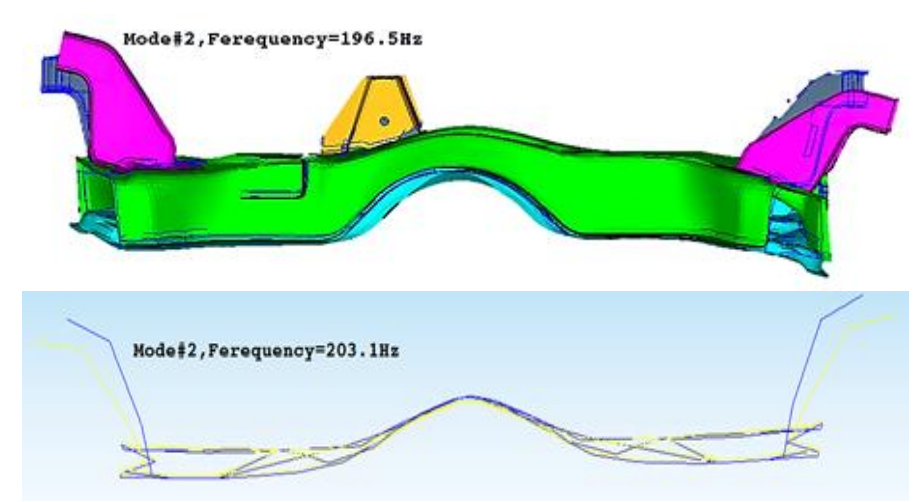

(b)
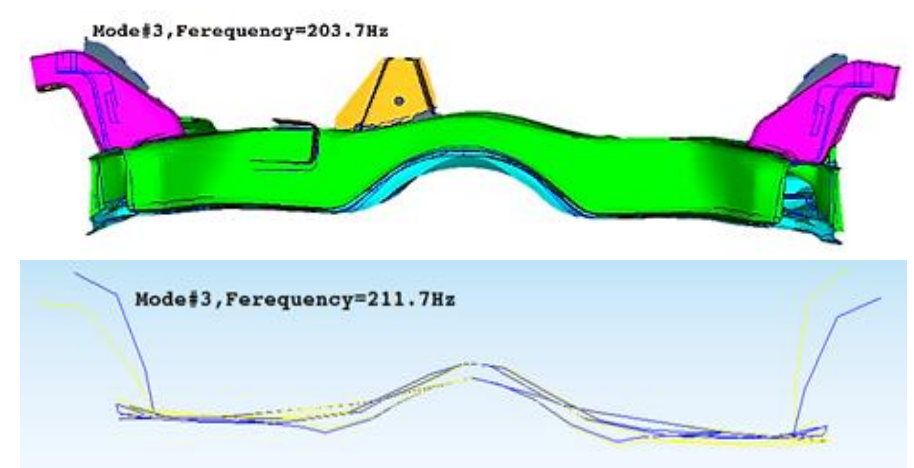

(c)
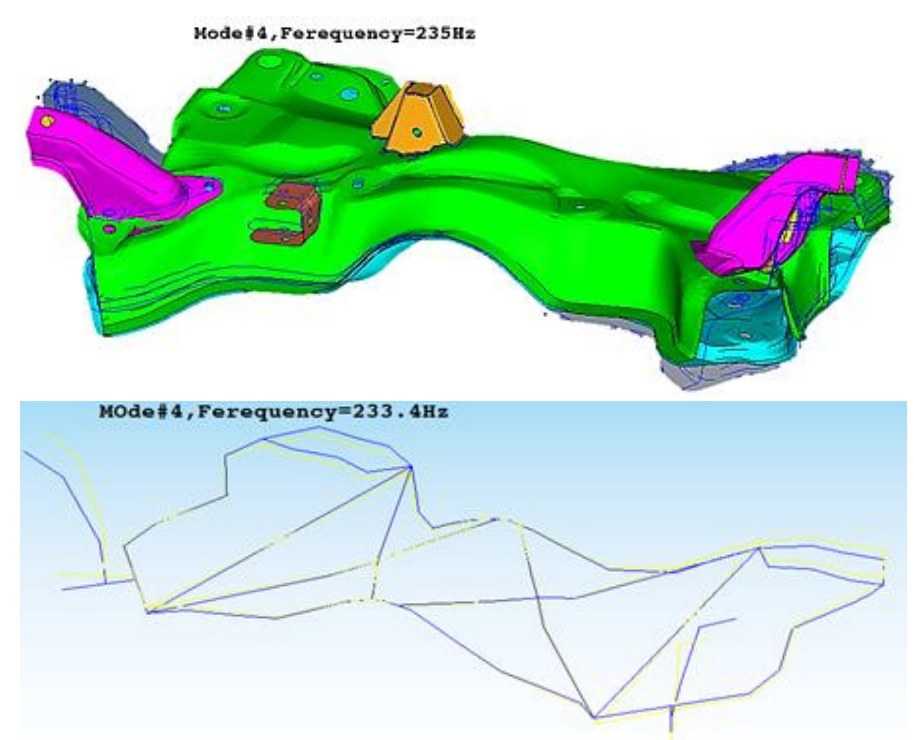

(d)

Figure 8. Subframe structural dynamics: (a) first, (b) second, (c) third and (d) fourth mode shapes of advanced and conceptual models.

\section{CONCLUSION}

The remarkable precision of the conceptual model presented in this study has the potential to provide sufficient accuracy in predicting and controlling the subframe's vibration in the frequency range of $0-300 \mathrm{~Hz}$. Although it is usually difficult to achieve a conceptual model 
at frequencies higher than $100 \mathrm{~Hz}$, it is made possible and thanks to the well-designed model. The results of the conceptual modelling of the vehicle subframe in comparison with the advanced engineering calculations model and its experimental test in the frequency range of $0-300 \mathrm{~Hz}$ illustrates the proper precision of the method is the difference between natural frequencies to be less than $8 \%$. It also provided reliable results for the modal assurance of the first and fourth modes with the value of above 0.9 and 0.7 for the second and third modes as the four primary and principal modes of the vehicle subframe. Also, the high match between the natural frequencies generated by the test with the CAE model paved the way for a conceptual model based on the CAD model.

It should be noted that due to the excessive reduction in the time of calculations for the conceptual model analysis compared with the advanced model (330 s vs $11.1 \mathrm{~s}$ ), the application of the method presented in this study in the design of the vehicle subframe significantly reduced the engineering time and improve the vibration performance of these structures. The sum of these periods in each structure and the various parts of the vehicle results in a great deal of time, which can be reduced by designing a more conceptual and straightforward model with the same behaviour prediction potential. Also, the lack of need for complete data to design subframe helps engineers to predict and improve its performance at an early stage. Due to the accuracy and applicability of the developed conceptual modelling method, this modelling method can be a significant strategy to manage noise and vibration for vehicle subframe structures. Therefore, using the results of the rapid changes in the model and analysing them, vehicle performance can be improved in the area of noise and vibration.

\section{ACKNOWLEDGEMENT}

This experimental work was supported by the Noise, Modal and Acoustic Laboratory at the University of Tehran.

\section{REFERENCES}

[1] Donders S, Takahashi Y, Hadjit R, Van Langenhove T, Brughmans M, Van Genechten B, et al. A reduced beam and joint concept modeling approach to optimize global vehicle body dynamics. Finite Elements in Analysis and Design. 2009;45:43955.

[2] Abdullah N, Sani M, Husain N, Rahman M, Zaman I. Dynamics properties of a gokart chassis structure and its prediction improvement using model updating approach. International Journal of Automotive \& Mechanical Engineering. 2017;14.

[3] Reed C. Applications of optistruct optimization to body in white design. In: Proceedings of Altair Engineering Event, Coventry, UK. 2002.

[4] Wang L, Basu PK, Leiva JP. Automobile body reinforcement by finite element optimization. Finite Elements in Analysis and Design. 2004;40:879-93.

[5] Gur Y, Wagner D. Damping properties and NVH modal analysis results of carbon fiber composite vehicle components. SAE International Journal of Materials and Manufacturing. 2017;10:198-205.

[6] Raasch I. Sizing in conceptual design at BMW. SAE Technical Paper 2004-01-1657, 2004.

[7] Zou T, Zhang J, Pang J, Li C, Liu B, Jia W, et al. The structure modal analysis and engineering application of the passenger car driver's seat system. In: Proceedings of SAE-China Congress 2015: Selected Papers: Springer; 2016. p. 349-63. 
[8] You YK, Yim HJ, Kim CM, Kim KC. Development of an optimal design program for vehicle side body considering the BIW stiffness and lightweight. SAE Technical Paper 2007-01-2357, 2007.

[9] Lee S-1, Lee D-c, Lee J-i, Han C-s, Hedrick K. Integrated process for structuraltopological configuration design of weight-reduced vehicle components. Finite elements in analysis and design. 2007;43:620-9.

[10] Fard M. Structural dynamics characterization of the vehicle seat for NVH performance analysis. SAE paper. 2011;501:2011.

[11] Sung SH, Nefske DJ. Assessment of a vehicle concept finite-element model for predicting structural vibration. SAE Technical Paper; 2001.

[12] Long L. Design-oriented translators for automotive joints. PhD thesis, Virginia Polytechnic Institute and State University, USA, 1998.

[13] Lee S, Park J, Yim H. Numerical approximation of vehicle joint stiffness by using response surface method. International Journal of Automotive Technology. 2002;3:117-22.

[14] Kiani M, Motoyama K, Rais-Rohani M, Shiozaki H. Joint stiffness analysis and optimization as a mechanism for improving the structural design and performance of a vehicle. Proceedings of the Institution of Mechanical Engineers, Part D: Journal of Automobile Engineering. 2014;228:689-700.

[15] Moroncini A, Cremers L, Kroiss M. NVH structural optimization using beams and shells FE concept models in the early car development phase at BMW. In: Proceedings of the International Conference on Noise and Vibration Engineering (ISMA'10), pp. 4163-4177; 2010.

[16] Mundo D, Donders S, Hadjit R, Stigliano G, Mas P, Van der Auweraer H. Concept modelling of automotive beams, joints and panels. In: Proceedings of World Scientific and Engineering Academy and Society (WSEAS) International Conference on FD, FEM, FV and BEM, Bucharest, Romania; 2010. p. 634-107.

[17] Mundo D, Hadjit R, Donders S, Brughmans M, Mas P, Desmet W. Simplified modelling of joints and beam-like structures for BIW optimization in a concept phase of the vehicle design process. Finite Elements in Analysis and Design. 2009;45:45662.

[18] Tebby S, Barari A, Esmailzadeh E. Optimization of vehicle structure considering torsion stiffness using simple structural beam frame-approach. Computer-Aided Design and Applications. 2013;10:413-26.

[19] Tebby S, Barari A, Esmailzadeh E. Development of optimized preliminary vehicle structural model using simple structural beams-frames (SSB) and sub-structural analysis. Computer-Aided Design and Applications. 2014;11:544-51.

[20] Marburg S, Hardtke H-J. Shape optimization of a vehicle hat-shelf: improving acoustic properties for different load cases by maximizing first eigenfrequency. Computers \& Structures. 2001;79:1943-57.

[21] Zhu P, Zhang Y, Chen G. Metamodel-based lightweight design of an automotive front-body structure using robust optimization. Proceedings of the Institution of Mechanical Engineers, Part D: Journal of Automobile Engineering. 2009;223:113347.

[22] Gündoğdu Ö. Optimal seat and suspension design for a quarter car with driver model using genetic algorithms. International Journal of Industrial Ergonomics. 2007;37:327-32. 
[23] Zargar O, Masoumi A, Moghaddam AO. Investigation and optimization for the dynamical behaviour of the vehicle structure. International Journal of Automotive and Mechanical Engineering. 2017;14:4196-210.

[24] Zargar O, Masoumi A, Ashoori MR. A conceptual model for the dynamical analysis of vehicle wheelhouse in the early stage of design. Modares Mechanical Engineering. 2016;15:279-86.

[25] Safaei B, Moradi-Dastjerdi R, Qin Z, Chu F. Frequency-dependent forced vibration analysis of nanocomposite sandwich plate under thermo-mechanical loads. Composites Part B: Engineering. 2018.

[26] Safaei B, Moradi-Dastjerdi R, Chu F. Effect of thermal gradient load on thermoelastic vibrational behaviour of sandwich plates reinforced by carbon nanotube agglomerations. Composite Structures. 2018;192:28-37.

[27] Hosseini R, Hamedi M, Golparvar H, Zargar O. Analytical and experimental investigation into increasing operating bandwidth of piezoelectric energy harvesters. AUT Journal of Mechanical Engineering. 2019;3:113-22.

[28] Azaripour S, Baghani M. Vibration analysis of FG annular sector in moderately thick plates with two piezoelectric layers. Applied Mathematics and Mechanics.1-22.

[29] Lo L, Fard M, Subic A, Jazar R. Structural dynamic characterization of a vehicle seat coupled with human occupant. Journal of Sound and Vibration. 2013;332:1141-52.

[30] Sun L, Chen N, Zhao Z. Experimental modal and dynamic performances analysis of car's body-in-white. Journal of Residuals Science \& Technology 2016;13.

[31] Safaei B, M Fattahi A. Free Vibrational Response of Single-Layered Graphene Sheets Embedded in an Elastic Matrix using Different Nonlocal Plate Models. Mechanics 2017;23:678-87.

[32] Azizi S, Safaei B, Fattahi A, Tekere M. Nonlinear vibrational analysis of nanobeams embedded in an elastic medium including surface stress effects. Advances in Materials Science and Engineering 2015;2015: 318539.

[33] Nastran M. Quick reference guide. MSC SOFTWARE. 2004;1.

[34] Shahriari B, Zargar O, Baghani M, Baniassadi M. Free vibration analysis of rotating functionally graded annular disc of variable thickness using generalized differential quadrature method. Scientia Iranica 2018;25:728-40.

[35] Jalali MH, Zargar O, Baghani M. Size-dependent vibration analysis of FG microbeams in thermal environment based on modified couple stress theory. Iranian Journal of Science and Technology, Transactions of Mechanical Engineering 2018;43(1):761.

[36] Hassen DB, Miladi M, Abbes MS, Baslamisli SC, Chaari F, Haddar M. Application of the operational modal analysis using the independent component analysis for a quarter car vehicle model. Advances in Acoustics and Vibration: Springer; 2017. p. 125-33. 